\title{
JET, THE LARGEST TOKAMAK ON THE EVE OF DT OPERATION
}

- L.D. Horton ${ }^{1,2}$ and the JET Contributors ${ }^{3}$ - DOI: http://dx.doi.org/10.1051/epn/2016503

- EUROfusion Consortium, JET, Culham Science Centre, Abingdon, OX14 3DB, UK

- ${ }^{1}$ JET Exploitation Unit, Culham Science Centre, Abingdon, OX14 3DB, UK

- ${ }^{2}$ European Commission, B-1049 Brussels, Belgium

- ${ }^{3}$ See the Appendix of F. Romanelli et al., Proceedings of the 25 $5^{\text {th }}$ IAEA Fusion Energy Conference 2014, St. Petersburg, Russia

\section{The Joint European Torus (JET) is the world's largest operating tokamak and the only such machine capable of operating with the fuel mixture (deuterium-tritium) foreseen for a fusion reactor. Since it came into operation in 1983, JET has explored fusion plasmas "in conditions and dimensions approaching those of a fusion reactor" [1]. JET has demonstrated world-record levels of fusion power and energy production, in conditions where the ratio of the fusion power generated to the input power to the plasma, $Q$, approaches unity.}

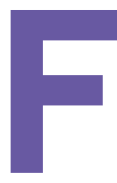

or the last decade, the JET has been executing a Programme in Support of ITER, the next-step device presently being built in the south of France (see article by D. Campbell). The cornerstone of this programme is the test of the interaction between fusion plasmas and ITER-relevant plasma-facing components (PFCs). To date, the majority of fusion experiments have used carbon as the material in these components because of carbon's tolerance to overheating. Carbon machines can test the boundaries of plasma performance in the knowledge that overloading the PFCs will not lead to changes in the component geometry. Carbon, on the other hand, interacts chemically with the fusion fuel, leading to a large fuel retention rate in the machine. This must be avoided in a fusion reactor due to the need to breed tritium in the process and on safety grounds. Indeed, both JET and ITER have strict limits on the maximum amount of tritium that can be trapped in the PFCs.

Carrying out JET's programme has required upgrades to the facility, in particular the installation of the same combination of plasma-facing materials planned for ITER (fig.1). In order to reach the highest fusion performance, JET's heating, diagnostic, protection and control systems have also been enhanced. The programme's primary objective is presently to develop techniques in deuterium plasmas of safely delivering the increased heating power to JET whilst maximising the plasma energy confinement and thus the equivalent fusion power and whilst respecting the power and energy limits of the new metallic PFCs.

Whilst the operating parameters of JET are the closest to those foreseen for ITER, there remains a considerable extrapolation between the two devices. The JET programme thus incorporates a very strong element of theory and model validation in order to provide a sound basis for this extrapolation.

Once safe and reliable operation in deuterium has been established, the last phase of JET's present programme is to test the dependence of this operation on the mass of the fuel ions and to study and optimise deuterium-tritium plasmas with large amounts of generated fusion power. These experiments will provide a unique operational and scientific knowledge base in preparation for ITER.

In addition to the plasma physics studies, a dedicated DT technology programme is underway with projects in the areas of neutron diagnostics and radiation damage, neutronics and activation code validation, the tritium cycle, and nuclear safety.

\section{Performance with}

the ITER wall material mix

Tungsten has ideal characteristics for the divertor targets, which are subject to the highest heat and particle fluxes: it has a high threshold for sputtering by plasma particles, the highest melting point of any metal, and an acceptably low affinity for hydrogen, implying a low rate of fuel retention. While the first wall is typically subject to much lower particle fluxes than the divertor, it is exposed to higher energy particles escaping from the core plasma. Since beryllium is a low- $Z$ material, beryllium atoms which penetrate the plasma after sputtering by these high-energy particles will contribute much less to plasma fuel dilution and plasma radiation than would tungsten. Beryllium's good thermal conductivity is also 
V FIG. 1: In any fusion experiment, managing the interaction between the hot plasma and the surrounding

plasma-facing surfaces is crucial. The solution foreseen for ITER, a combination

of beryllium in

the main vacuum chamber near the hot fusion plasma and tungsten facing the lower temperature divertor plasma (at the bottom of the machine), is now being tested at JET. (c) CPS15.139-2C

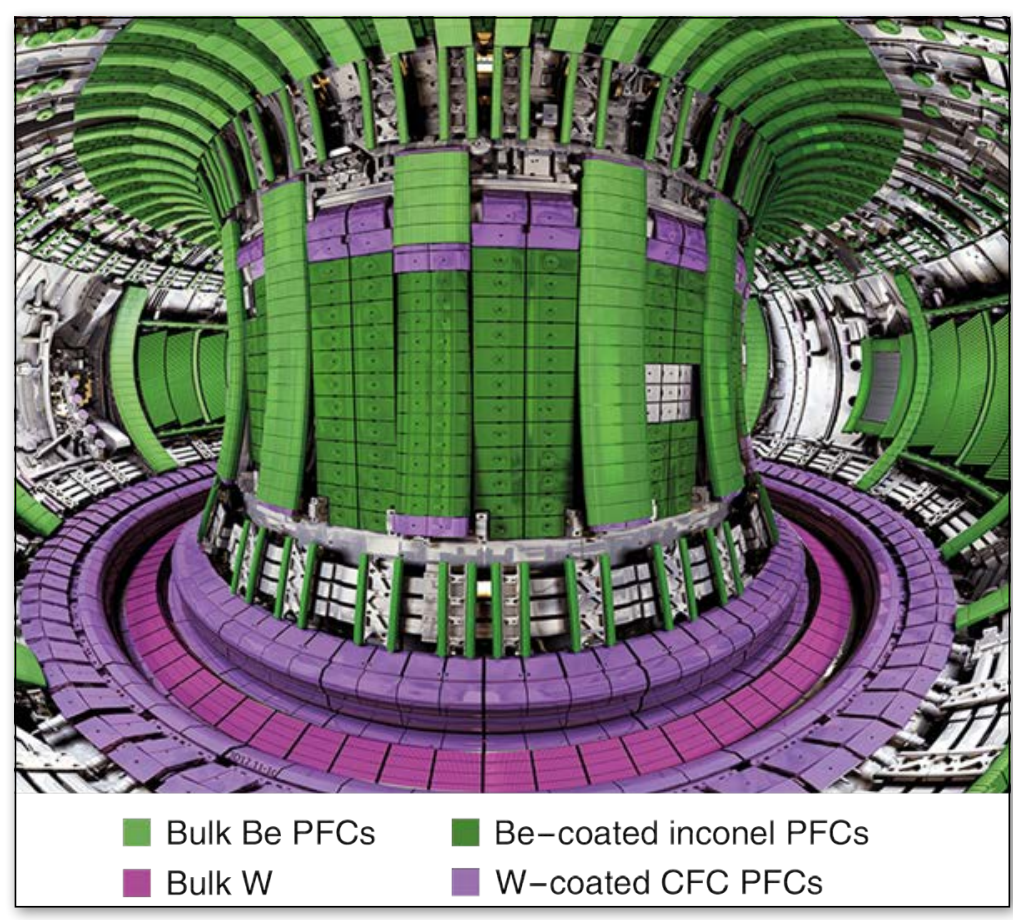

advantageous in this application. Nevertheless, there is a residual risk of localised melting of both beryllium and tungsten under the high transient heat loads which can occur during sudden plasma events such as 'disruptions'. Adequate mitigation measures must therefore be in place to dissipate the plasma energy losses that can occur in such cases, and this is a major focus of current fusion plasma research.

The retention of hydrogen isotopes in JET has been measured both before the installation of the new wall, when JET's plasma-facing components were predominantly made of carbon, and with the new beryllium-tungsten components. The expected reduction in fuel retention of more than an order of magnitude has been confirmed, a very positive result for ITER. More importantly, the codes that describe the processes of wall erosion, material migration and re-deposition have been benchmarked on JET, also to the level of understanding the spatial pattern of these processes inside the machine. This has greatly increased confidence that the predictions of fuel retention being made for ITER are accurate.

With the change in wall material, it has been necessary to re-optimise the fusion plasmas in order to respect the tighter power and energy limits on the metallic PFCs and because of the potentially large impact of the new materials on the plasma energy confinement. A good example is the need to manage the source and transport of tungsten from the divertor to the hot fusion plasma. Accumulation of tungsten in the plasma core can lead to strong radiative losses that more than compensate for central heating and thus to hollow temperature profiles (fig.2). Control of the tungsten transport by the use of central heating can be used to avoid such accumulation and recover the high temperature conditions.

\section{Experiments with Tritium}

Tritium affects the physics of fusion plasmas via its increased mass and, when used in combination with deuterium, via the production of high-energy alpha particles from the D-T fusion reaction. The fuel mass influences particle and energy transport due to the change in ion gyro-radius and is important also for heating schemes based on the ion cyclotron resonance (ICRH). Alpha particle production is ultimately the scheme that will be used in ITER to sustain the plasma temperature. Indeed, understanding the additional dynamics generated by such a self-sustained or burning plasmas is a key scientific objective for ITER. In JET, the power deposited in the plasma by fusion alphas will always be a small fraction of the total heating power and thus the alpha particle physics on JET focuses on single particle and threshold effects related to fast particle - wave interactions.

\section{Integration and Performance Optimisation}

Two routes are being explored to bring JET to maximum fusion performance. The first relies on the fact that the energy confinement of the plasma increases with plasma current. By operating at the maximum possible plasma current, it was possible in the first high power DT experiment in 1997 to generate $4 \mathrm{MW}$ of fusion power for the duration of the high power heating phase.

Since the experiments in 1997, it has been realised that operation at high plasma pressure can provide access to higher confinement and thus higher fusion performance for the same plasma current. This improvement with plasma pressure is then limited by the plasma stability. The second route to high fusion performance on JET relies on achieving the maximum safe plasma pressure at somewhat reduced plasma current.

Both routes to maximum fusion performance depend on applying the full available heating power and therefore on managing the exhaust of that level of power. Techniques including stability control and mitigation, seeding of extrinsic impurities to enhance edge radiation and sweeping of the areas of maximum plasma load across a wider region of the wall are being developed. The goal is to achieve fusion power well above the $4 \mathrm{MW}$ reached in 1997. Obtaining standard confinement, as defined in the scaling laws used to design ITER, at JET's highest current is predicted to lead to a fusion power of about 10 MW. Matching or even bettering this target is the goal of the research into the high plasma pressure optimisation.

\section{DT Technology Programme}

The use of tritium and the production of large amounts of $14 \mathrm{MeV}$ neutrons provide a unique opportunity to validate codes, assumptions, models, procedures and data currently used for ITER.An important example is the benchmarking of neutronics codes for the calculation of neutron streaming 


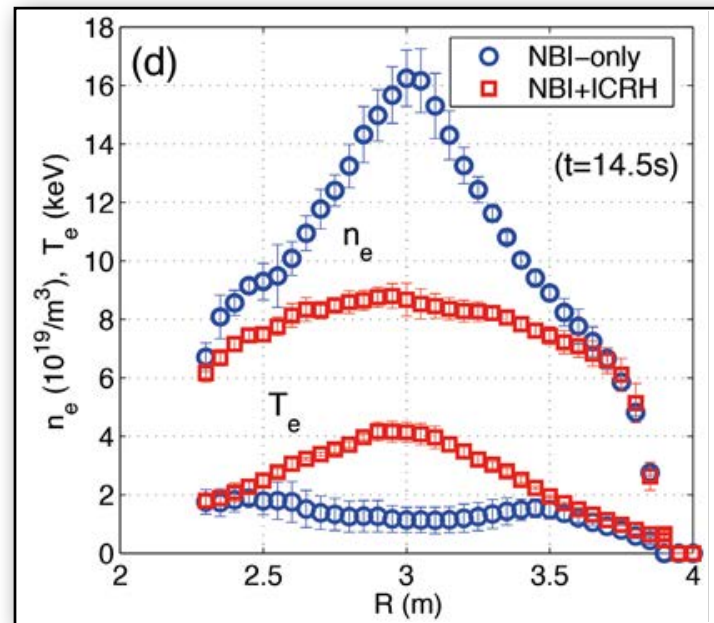

through penetrations in the JET biological shield and for subsequent evaluation of the gamma dose rates in remote areas. Maps of the predicted neutron fluence generated by the planned high power DT experiment are given in fig.3. Measurements to validate these calculations are planned using a combination of activation foils and thermo-luminescent dosimeters. This validation will support the development of maintenance activities on ITER.

\section{Conclusion}

The planned extensive use of tritium in JET supports an important transition in the European fusion research programme towards the realisation of a nuclear tokamak and of fusion electricity on the grid [4]. It is an explicit strategic goal of the European fusion programme (see article by $\mathrm{T}$. Donné) to train the scientists and engineers who will run ITER and JET plays a key role in achieving that goal.

The JET experiments are logically divided in two; a first Isotope Experiment in which plasma performance will be compared for all three hydrogen isotopes (protium, deuterium and tritium) and a subsequent Deuterium-Tritium Experiment in which high fusion yields will be produced and the physics associated with fusion alpha particles will be studied. On the present schedule the Isotope Experiment will take place in 2018-19 and the DT Experiment in 2019-20. Together and based on previous experimentation with JET's ITER-like Wall, these experiments allow optimisation of the ITER research plan and, in particular, for the transition from protium to deuterium to deuterium-tritium plasmas that will take place as ITER moves from commissioning to nuclear operation.

\section{Acknowledgement}

This work has been carried out within the framework of the EUROfusion Consortium and has received funding from the Euratom research and training programme 2014-2018 under grant agreement No 633053. The views and opinions expressed herein do not necessarily reflect those of the European Commission.
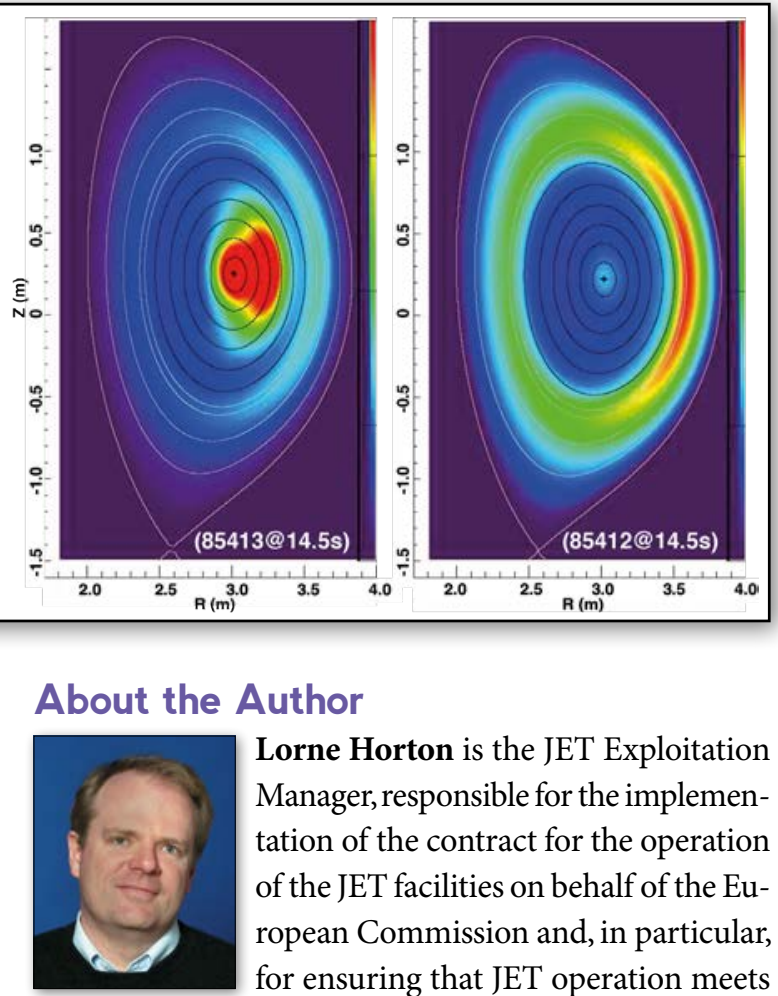

Lorne Horton is the JET Exploitation Manager, responsible for the implementation of the contract for the operation of the JET facilities on behalf of the European Commission and, in particular, for ensuring that JET operation meets the needs of the JET scientific programme defined by the EUROfusion consortium of EU fusion laboratories.

\section{References}

[1] The JET Project (Design Proposal), Report of the Commission of the European Communities, EUR 5516e, 1976.

[2] E. Lerche, M. Goniche, P. Jacquet, et al., Nucl. Fusion 56, 036022 (2016) (19pp).

[3] P. Batistoni, D. Campling, S. Conroy et al., Fusion Eng. Des. 109111, 278 (2016).

[4] F. Romanelli, P. Barabaschi, D. Borba, et al., A roadmap to the realisation of fusion energy, EFDA Report, ISBN 978-3-00040720-8 and https://www.euro-fusion.org/eurofusion/ the-road-to-fusion-electricity/

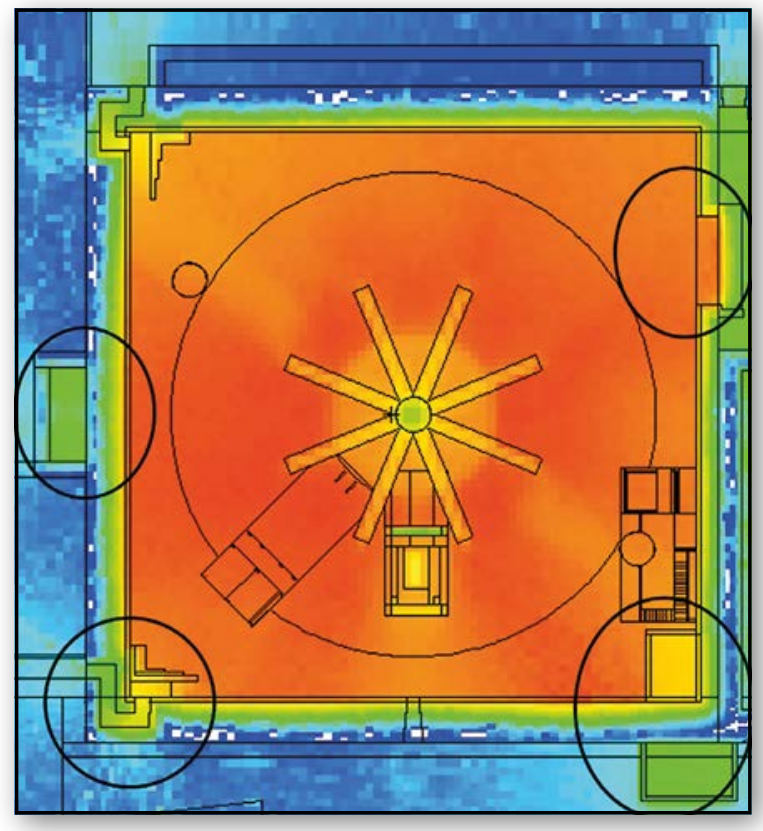

4 FIG. 2: Accumulation of tungsten in the centre of the fusion plasma can lead to large radiative losses (centre panel) central peaking of the plasma density and even inverted temperature profiles. The addition of central electron heating, in this case using ion cyclotron resonance heating (ICRH) can be used to control the tungsten transport in the plasma, avoiding the central radiation (right panel) so that the hot fusion conditions are recovered (from [2]).

FIG. 3: Neutron fluence maps for the planned deuteriumtritium experiment, highlighting the areas in which neutron streaming through penetrations in the JET torus hall will be measured. Benchmarking these calculations with measurements will increase confidence in planning maintenance activities in ITER (from [3]). 